\title{
Behandlungsfehler, Patientensicherheit und Patientenrechtegesetz - ein Spannungsfeld, das uns alle angeht
}

\author{
Treatment Errors, Patient's Safety and Patient's Legal Rights - An Area of Tension that Concerns Us All
}

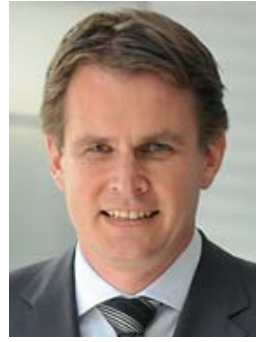

U. Stöckle

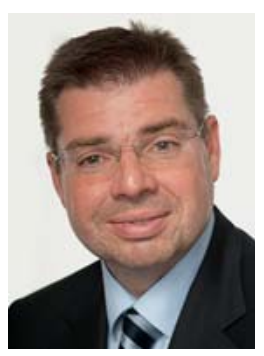

D. C. Wirtz

\section{Bibliografie}

DOI http://dx.doi.org/

$10.1055 / \mathrm{s}-0032-1327826$

Z Orthop Unfall 2012; 150: 461-

462 @ Georg Thieme Verlag KG Stuttgart · New York .

ISSN 1864-6697

\section{Korrespondenzadressen Univ.-Prof. Dr. Ulrich Stöckle Ärztlicher Direktor \\ BGU Klinik Tübingen Schnarrenbergstraße 96 72076 Tübingen \\ Tel.: 0 07071/606-1001 Fax: 07071/606-1002 ustoeckle@bgu-tuebingen.de}

\section{Univ.-Prof. Dr. med}

Dieter C. Wirtz

Direktor der Klinik und Poliklinik für Orthopädie und Unfallchirurgie Universitätsklinikum Bonn Sigmund-Freud-Straße 25 53105 Bonn

Tel.: 0228/287-14170

Fax: 0228/287-14175

dieter.wirtz@ukb.uni-bonn.de

\section{Liebe Leserinnen und Leser, $\nabla$}

durch die Einführung des Patientenrechtegesetzes, über das im Bundestag voraussichtlich noch im Laufe des Jahres beraten wird, soll es dem Patienten leichter gemacht werden, sich gegen Behandlungsfehler zu wehren. Grundsätzlich ist dieser Weg hin zur Etablierung einer Patientensicherheitskultur zu begrüßen. Dabei darf es aber nicht um die ausufernde Infragestellung jeglicher nicht optimaler Behandlungsergebnisse gehen. Gerade den Kostenträgern, die mit dem Gesetz verpflichtet werden sollen, jedem Vorwurf ihrer Mitglieder nachzugehen, kommt dabei ein hohes Maß an Verantwortung zu. Die Differenzierungsfrage zwischen einem anzuerkennenden Behandlungsfehler und einer unvermeidbaren Komplikation wird sicherlich häufiger als bisher die Gutachterkommissionen und Schlichtungsstellen der Ärztekammern wie auch die Medizinischen Dienste der Krankenkassen (MDK) beschäftigen. Entsprechend der Behandlungsfehlerstatistik der MDK-Gemeinschaft bearbeiteten die MDK-Gutachter im Jahr 2011 insgesamt 12686 Behandlungsfehlervorwürfe; im Jahr 2007 waren es noch weniger als 11000 (Quelle: www.aerzteblatt.de/ nachrichten [5. Sept. 2012]). Dabei blieb die Anerkennungsquote allerdings mit ca. $30 \%$ weitgehend unverändert - insgesamt wurden 4068 der Behandlungsfehlervorwürfe als gerechtfertigt angesehen. Unser Fach Orthopädie und Unfallchirurgie steht hier - leider - im besonderen Fokus: unter den 10 häufigsten Krankheitsbildern, bei denen Behandlungsfehler bestätigt wurden, sind allein 6 aus unserem Gebiet zu finden. Die Kniearthrose (159) und die Hüftarthrose (140) liegen auf Platz 1 und 2, gefolgt von Zahnkaries (134), Oberschenkelbruch (111), Zahnnerventzündung (108), Unterschenkelbruch (85), Dekubitus (81), sonstige Zahnkrankheiten (73), Unterarmbruch (67) und Bandscheibenschäden (58) (Quelle: Medizinischer Dienst des Spitzenverbands Bund der Krankenkassen [MDS], www.mds-ev.de)). Geht man der Frage nach, wie hoch die Anerkennungsquote in Bezug auf die Antragsstellungen liegt, so liegt die Orthopädie und Unfallchirurgie mit 30\% ebenfalls mit Patz 4 relativ weit vorne, hinter der Pflege (51\%), Zahnmedizin (43\%) und Gynäkologie (34\%).

Auch wenn man der gutachterlichen Bewertung des MDK entgegenhalten kann, dass nicht immer das Prinzip der Facharztgleichheit eingehalten wird, so decken sich die genannten MDK-Zahlen doch weitgehend mit den Statistiken der Schlich- tungsstellen der Ärztekammern. Von den in 2011 bearbeiteten 7500 Fällen wurden etwa ein Drittel als Behandlungsfehler anerkannt. Nach Angabe der Ärztekammer Nordrhein entfielen in 2011 von insgesamt 520 anerkannten Fällen 89 (31,6\%) auf das Fachgebiet Orthopädie und Unfallchirurgie (Quelle: Bericht der Gutachterkommission für ärztliche Behandlungsfehler der Ärztekammer Nordrhein 19.11.2011).

Aufgrund dieser Datenlage sind wir als Orthopäden und Unfallchirurgen ganz besonders gefragt, die „Fehlervermeidung“ als integralen Bestandteil unseres täglichen Handelns anzusehen. Fehler dürfen dabei nicht nur als Fehlverhalten Einzelner aufgefasst werden, sondern vielmehr als Versagen im System. Ein System, das zunehmend geprägt wird von Arbeitsverdichtung, Ökonomisierung und Arbeitsdelegation, muss kritisch hinterfragt und gegebenenfalls verändert werden. Es gilt daher herauszufinden, wo und weshalb sich Fehler häufen und wie diese künftig vermieden werden können. Unser Fach wird sich künftig ganz besonders und intensiv den Fragen der Struktur- und Qualitätsverbesserung stellen müssen. Diese Aufgabe wollen und müssen wir angehen. Deshalb ist es aber auch wichtig, sich nicht nur auf der rein medizinischen Ebene zu bewegen. Der fachverantwortliche Arzt muss in Klinik und Praxis mit einem entsprechenden Wissen und Verantwortungsbewusstsein sowohl die Struktur- als auch Organisationsdirektive besitzen. Wenn von Einkäufern oder Einkaufsverbünden aufgrund ausschließlich wirtschaftlicher Überlegungen die zu verwendenden Implantate vorgeschrieben werden, diese aber möglicherweise mit einer höheren Versagerquote versehen sind (wie bei der aktuellen Diskussion bei bestimmten Metall-Metall-Gleitpaarungen evident), so darf berechtigterweise die Frage gestellt werden: wer ist verantwortlich für den beim Patienten entstandenen Schaden?

Auch ein weiterer Aspekt wird uns in diesem Zusammenhang künftig immer mehr beschäftigen: da es in vielen Kliniken durch das Gebot der Wirtschaftlichkeit zunehmend zur Neuverteilung von Aufgaben kommt, sich neue Gesundheitsfachberufe etablieren und die Profile der Berufsgruppen verschwimmen, entsteht die Frage, wer die Organisationsverantwortung trägt. Während Ärzte weiterhin kodieren, Briefe schreiben, Reha-Anträge stellen und Krankenkassenanfragen beantworten, stehen zunehmend Nichtärzte im OP. Meist gehören diese jedoch unter die Personalhoheit der Pflegedirektion. Auch wenn das konkret 
durchgeführte Handeln einer auch rechtlich eindeutig zu bewertenden ärztlichen Delegationsleistung entspricht, wird es doch immer schwieriger, gerade für dieses „den Arzt entlastenden Personal“ die Organisationsverantwortung zuzuordnen und letztendlich zu übernehmen.

Wir als Orthopäden und Unfallchirurgen wollen und sollen diese vielfältigen Verantwortungsbereiche mittragen bzw. beibehalten. Patientensicherheit und ergebnisorientierte Behandlungsqualität müssen oberste Priorität besitzen. Das Unternehmen Krankenhaus bedarf der ethischen Verantwortung des Arztes. Ihm darf auch mal ein Fehler unterlaufen, aber durch eine möglichst optimale Organisations- und Weiterbildungsstruktur sollte alles dafür getan werden, solche zu vermeiden. Fehlervermeidung bedeutet daher stetige und durchaus selbstkritische Hinterfragung des eigenen Handelns, aber auch die aktive Mitgestaltung des täglichen - und insbesondere dem Wirtschaftlichkeitsgebot unterliegenden - Arbeitsumfelds.

Deshalb ist es wichtig, die verschiedenen Qualitätsinitiativen unserer Fachgesellschaften, wie z.B. die Etablierung von Trauma- netzwerk und Endoprothesenregister, fest in die Versorgungsstruktur unseres Gesundheitssystem zu integrieren. Auch wenn Zertifizierungsmaßnahmen zur Bildung von Trauma- und Endoprothesenzentren kosten- und zeitaufwendig sind und von dem ein oder anderen der Kritik der „Zertifizitis“ unterzogen werden, so tragen diese Strukturmaßnahmen sicherlich zur verbesserten Versorgungsqualität und Patientensicherheit bei. Jedes Krankenhaus, welches die geforderten Voraussetzungen in personeller und struktureller Hinsicht erfüllt, soll und darf das Qualitätssiegel des „zertifizierten Zentrums“ tragen. Kliniken, die ihre Schwerpunktausrichtung in anderen Struktur- oder Fachbereichen sehen, müssen dies akzeptieren und damit auch ehrlich umgehen. Das schafft Vertrauen zwischen Klinik, Arzt und Patient. Ein Gut, das wir künftig pflegen sollten und müssen.

Ihre

Prof. Dr. med. Ulrich Stöckle Prof. Dr. med. Dieter C. Wirtz 